\title{
STABILITY AND ALMOST PERIODICITY IN DYNAMICAL SYSTEMS ${ }^{1}$
}

\author{
C. R. PUTNAM
}

1. In the system of differential equations

$$
x^{\prime}=f(x)
$$

$\left({ }^{\prime}=d / d t\right)$,

let $x=\left(x_{1}, \cdots, x_{n}\right)$ and $f=\left(f_{1}, \cdots, f_{n}\right)$, and suppose that $f$ is of class $C^{1}$. A set, $\Omega$, of points $x$ will be called unrestricted if, whenever a point $x_{0}$ is in $\Omega$, the solution path $x=x(t)$, where $x_{0}=x(0)$, exists for $-\infty<t<\infty$ and lies in $\Omega$. Two types of stability distinguished simply by "A" and "B" will be considered:

(A) Let the value $t_{0}$ be arbitrary but fixed. A solution $x=x(t)$ of (1) will be called $A$-stable with respect to an unrestricted (hence invariant) set, $\Omega$, if $x(t)$ is in $\Omega$ and if there exists for every $\epsilon>0$ a $\delta$ $=\delta_{\epsilon}>0$ such that $|x(t)-y(t)|<\epsilon,-\infty<t<\infty$, whenever $y(t)$ is in $\Omega$ and $\left|x\left(t_{0}\right)-y\left(t_{0}\right)\right|<\delta$.

(B) A solution $x=x(t)$ of (1) will be called $B$-stable with respect to an unrestricted set, $\Omega$, if $x(t)$ is in $\Omega$ and if there exists for every $\epsilon>0$ a $\delta=\delta_{\epsilon}>0$ such that $|x(t)-y(t)|<\epsilon$ whenever $y(t)$ is in $\Omega$ and $\left|x\left(t_{0}\right)-y\left(t_{0}\right)\right|<\delta$ holds for some value $t=t_{0},-\infty<t_{0}<\infty$.

Of course, in both types of stability, the number $\delta$ depends not only on $\epsilon$ but also on the particular solution $x(t)$ considered. The notion of $A$-stability is that associated with Minding, Dirichlet (in the case of equilibrium solutions), and Liapounoff $[\mathbf{5}$, pp. 98-99; 3, pp. 210-211]. The $B$-stability was considered by Hartman and Wintner [1].

It is easy to see that a solution which is $B$-stable is surely $A$-stable. The converse is false however. In fact, one need only consider the single differential equation $(n=1)$

$$
x^{\prime}=\cos ^{2} x,
$$

and let $\Omega$ denote the set $-\pi / 2 \leqq x \leqq \pi / 2$. Then the equation (2) has the solutions $x=\pi / 2, x=-\pi / 2, x=\operatorname{Arc} \tan \left(t-t^{*}\right)$, where $t^{*}$ is arbitrary; clearly, $\Omega$ is a compact, unrestricted, invariant set of (2). It is easy to see that any solution $x=x(t)=\operatorname{Arc} \tan \left(t-t^{*}\right)$ is $A$-stable with respect to $\Omega$. (Note that the number $t=t_{0}$ occurring in the definition of $A$-stability is arbitrary but fixed.) On the other hand, it is clear

Received by the editors November 4, 1953.

1 This research was supported, in part, by the National Science Foundation research grant NSF-0101. 
that no solution of (2) is $B$-stable; the confluence property of the solutions, namely that $x(t) \rightarrow \pi / 2(-\pi / 2)$ when $t \rightarrow \infty(-\infty)$, precludes this type of stability. In fact, it is clear that a necessary condition in order that a function $x(t)$ be $B$-stable with respect to a set $\Omega$ is that $\lim \inf |y(t)-x(t)|>0$, as $t \rightarrow \infty$, holds for every function $y(t)(\not \equiv x(t))$ in $\Omega$.

It was shown by Hartman and Wintner [1] that if $\Omega$ is the closure of the path $x=x(t)$, and if $\Omega$ is compact, then $x=x(t)$ is $B$-stable with respect to $\Omega$ if and only if $x(t)$ is almost periodic (in the sense of Bohr), in which case, every solution $y=y(t)$ contained in $\Omega$ is almost periodic. However, the assumption of $B$-stability is rather stringent as compared with the classical type of $A$-stability. That $A$-stability alone, though, is not sufficient to guarantee almost periodicity is clear from a consideration of the solutions $x(t)=\operatorname{Arc} \tan \left(t-t^{*}\right)$ of (2). (Note that each of these solutions $x(t)$ is dense on the space $\Omega:-\pi / 2 \leqq x$ $\leqq \pi / 2$.)

In the considerations below, the set $\Omega$ will denote a compact, invariant (hence unrestricted) set not necessarily restricted to the case that it be the closure of the particular solution path in question.

It is interesting to note that the Poincaré-Birkhoff criterion for the stability of equilibrium solutions, involving a sequence of invariant sets, relates to $A$-stability.

In the case of conservative dynamical systems, an extra condition (incompressibility) is also satisfied, namely

$$
\operatorname{div} f \equiv \sum_{k=1}^{n} \partial f_{k} / \partial x_{k}=0 .
$$

(Of course, a system (1) may satisfy (3) even if it is not of the dynamical type.) The significance of the condition (3) is simply that of preservation of (volume) measure; thus, meas $S_{t}=$ meas $S_{0}$, where $S_{t}=T^{t}\left(S_{0},\right) x(t)=T^{t}\left(x_{0}\right)\left(x(0)=x_{0}\right)$, and $S_{0}$ is an arbitrary measurable set of points $x_{0}$.

It should be pointed out that the only known $A$-stable solutions $x=x(t)$, other than equilibrium solutions, in the case of dynamical systems (1) are almost periodic; cf. [1, p. 273]. If the space $\Omega$ is compact and identical with the closure of $x(t)$, then the HartmanWintner result (valid even if (3) does not hold) implies that $x(t)$ is even $B$-stable with respect to $\Omega$.

Whether, in the dynamical case, every solution $x=x(t)$ of (1), which is $A$-stable with respect to some compact invariant set (not necessarily equal to the closure of $x(t)$ ), is necessarily almost periodic will remain undecided. It will turn out that, although almost periodicity 
is not claimed in this case, still the three italicized assertions of $\$ 2$ below hold. Each of these properties is, in fact, possessed by an almost periodic function. (In connection with $(* * *)$, see $[4$, p. 21].)

2. Let now $\Omega$ be an unrestricted, invariant set of (1) of finite positive (volume) measure and suppose that (3) holds (so that the measure is preserved). The present set-up is essentially that specified for the application of the Poincare Recurrence Theorem or the Birkhoff Ergodic Theorem (both of which exempt from their claims certain sets of measure zero); cf., e.g., [5, pp. 89, 91].

According to the Poincare theorem, if $x=x(t)$ denotes a solution of (1) passing through a fixed point $x_{0}$ of $\Omega$, then for almost all points $x_{0}$ of $\Omega$, the following holds: If $x(t)$ attains a value $x\left(t_{0}\right)$ at a time $t_{0}$, then there exists an infinity of dates, tending to infinity both in the past and in the future, at which the path $x(t)$ comes arbitrarily close to the point $x\left(t_{0}\right)$. Due to the "almost everywhere" nature of the assertion, there exists an amount of indefiniteness ("to within a zeroset") of the validity of the assertion for a specific point $x_{0}$ of the space $\Omega$. However, if the point $x_{0}$ through which the given solution $x=x(t)$ passes is an $A$-stable point (that is, if $x(t)$ is a solution of (1) which is $A$-stable with respect to $\Omega$ ), and provided that $\Omega$ is suitably restricted, then with respect to the particular path $x=x(t)$ the Poincaré theorem can be refined as follows:

${ }^{*}$ ) Let $\Omega$ be a compact, invariant set of the system (1) with a (necessarily finite) positive measure and suppose that the measure-preserving condition (3) holds. In addition, suppose that the product set $\Omega \Sigma_{x}$ has a positive measure whenever $x$ is an arbitrary point of $\Omega$ and $\Sigma_{x}$ is an arbitrary $n$-sphere with center at $x$. If $x=x(t)$ is an $A$-stable solution of (1), and if $t=t_{0}$ is arbitrary, then there exists a sequence of dates, $t_{n}$, where $n= \pm 1, \pm 2, \cdots$, such that $t_{n} \rightarrow \infty$ or $-\infty$ according as $n \rightarrow \infty$ or $-\infty$, and $x\left(t_{n}\right) \rightarrow x\left(t_{0}\right)$ as $|n| \rightarrow \infty$.

The assumption of $\left({ }^{*}\right)$ relating to the sets $\Omega \Sigma_{x}$ will surely be satisfied in case the set $\Omega$ is, for instance, the closure of the set of points $\sum S_{t}$, $-\infty<t<\infty$, where $S_{t}=T^{t}\left(S_{0}\right), x(t)=T^{t}\left(x_{0}\right)$, and $S_{0}$ is an arbitrary open set of points $x_{0}$.

It will be clear from the proof given below that the assertion of $\left(^{*}\right)$ remains valid if the assumption that $\Omega$ be compact is replaced by the requirement that $\Omega$ be a set of finite positive measure. (The compactness property of $\Omega$ will be used in (**) and (***) however.) It should be noted that the assertion of $\left(^{*}\right)$ may fail to hold if the assumption (3) is dropped. This follows from a consideration of the system (2) in which $x(t)$ denotes any of the $A$-stable solutions Arc $\tan \left(t-t^{*}\right)$ and $\Omega$ denotes the interval $-\pi / 2 \leqq x \leqq \pi / 2$. 
In order to prove $\left(^{*}\right)$, suppose, if possible, the contrary. That is, suppose that there exists a date $t=t_{0}$ and an $\epsilon>0$ such that

$$
\left|x(t)-x\left(t_{0}\right)\right|>3 \epsilon \quad \text { for all } t>\text { const. }
$$

(For simplicity, only values $t \rightarrow \infty$ will be considered; the argument for $t \rightarrow-\infty$ is similar.) Since $x(t)$ is $A$-stable, there exists a $\delta=\delta_{e}>0$ such that

$$
|x(t)-y(t)|<\epsilon,-\infty<t<\infty \text {, whenever }|x(0)-y(0)|<\delta .
$$

Consider the set of all points $y_{0}=y(0)$ lying inside a fixed sphere $\Sigma$ of radius $\delta$ about the point $x(0)$; then, in view of (4) and (5), one has $\left|y(t)-y\left(t_{0}\right)\right|>\epsilon$ for all sufficiently large $t$. Thus every point of $\Omega \Sigma$ fails to satisfy the assertion of the Poincare Recurrence Theorem. Since, by assumption, the set $\Omega \Sigma$ has positive measure, a contradiction to the Poincaré theorem is obtained and so the proof of $\left(^{*}\right)$ is complete.

A theorem analogous to $\left(^{*}\right)$ but which is related to the Birkhoff Ergodic Theorem can be stated as follows:

$\left({ }^{* *}\right)$ Let $\Omega$ satisfy the same conditions as in $\left({ }^{*}\right)$ and let $g=g(x)$ denote any continuous function on $\Omega$. If $x(t)$ is $A$-stable with respect to $\Omega$ then

$$
\lim _{T \rightarrow \infty} T^{-1} \int_{0}^{T} g(x(t)) d t \text { exists. }
$$

(Needless to say, the relation (6) is not invalidated if $\infty$ is replaced by $-\infty$.)

The proof of $\left({ }^{* *}\right)$ is similar to that of $\left(^{*}\right)$. Let $x_{0}$ be a fixed point of the $A$-stable path $x(t)$ and put $L\left(x_{0}\right)=\lim$ sup $T^{-1} \int_{0}^{T} g(x(t)) d t$, $l\left(x_{0}\right)=\lim$ inf $T^{-1} \int_{0}^{T} g(x(t)) d t$, as $T \rightarrow \infty$. Suppose, if possible, that $L\left(x_{0}\right)-l\left(x_{0}\right)>0$. Since $\Omega$ is compact, $g=g(x)$ is uniformly continuous on $\Omega$, so that $|g(x+\Delta x)-g(x)|<\epsilon$ if $|\Delta x|<\delta_{\epsilon}(x, x+\Delta x$ arbitrary points of $\Omega$ ). Since $x(t)$ is $A$-stable, $y(t) \rightarrow x(t)$ uniformly in $t,-\infty<t$ $<\infty$, as $y_{0} \rightarrow x_{0}$. (Here, if $x\left(t_{0}\right)=x_{0}$, then $y(t)$ denotes a solution of (1) such that $y\left(t_{0}\right)=y_{0}$.) Consequently, both limit relations $g(y(t))$ $\rightarrow g(x(t))$, uniformly in $t$ on $-\infty<t<\infty$, and

$$
T^{-1} \int_{0}^{T} g(y(t)) d t \rightarrow T^{-1} \int_{0}^{T} g(x(t)) d t,
$$

uniformly in $T$ on $-\infty<T<\infty \quad(T \neq 0)$, hold as $y_{0} \rightarrow x_{0}$. Hence $L\left(y_{0}\right)$ $-l\left(y_{0}\right)>0$ for all $y_{0}$ in the set $\Omega \Sigma$. Since the set $\Omega \Sigma$ has a positive measure, a contradiction is obtained to the Birkhoff theorem. In fact, 
$g(x)$ is surely of class $L$ on $\Omega$ and so (6) must hold for almost all paths $x=y(t)$, that is, for almost all points $y_{0}$ of $\Omega$. This completes the proof of $(* *)$.

It is interesting to consider the particular case of $(* *)$ in which $g(x)=x_{k}^{m}$, where $x=\left(x_{1}, \cdots, x_{n}\right)$ and $m$ is a positive integer, so that $g(x)$ denotes the $m$ th power of the $k$ th coordinate of the point $x$. Clearly $g(x)$ is continuous on $\Omega$ so that, if $x(t)$ is stable, then all the limits

$$
\lim _{T \rightarrow \infty} T^{-1} \int_{0}^{T} x_{k}^{m}(t) d t, \quad m=1,2, \cdots,
$$

exist. Since $x_{k}(t)$ is bounded on $\Omega$, this implies (cf. [2, p. 76]) the following result:

$\left({ }^{* *}\right)$ If $\Omega$ satisfies the same conditions as in $\left(^{*}\right)$ and if $x=x(t)$ is $A$-stable with respect to $\Omega$, then $x(t)$ possesses an asymptotic distribution function.

Thus, there exists a distribution function $\phi=\phi(E)$ defined on the Borel sets $E$ of $\Omega$ such that $\phi_{T}(E) \equiv T^{-1}$ meas $\{t$ on $[0, T] ; x(t)$ in $E\}$ $\rightarrow \phi(E)$, as $T \rightarrow \infty$, provided $E$ is a continuity set of $\phi ;$ cf. [1, pp. 277$278]$.

\section{REFERENCES}

1. P. Hartman and A. Wintner, Integrability in the large and dynamical stability, Amer. J. Math. vol. 65 (1943) pp. 273-278.

2. B. Jessen and A. Wintner, Distribution functions and the Riemann zeta-function, Trans. Amer. Math. Soc. vol. 38 (1935) pp. 48-88.

3. A. Liapounoff, Problème gênéral de la stabilité du mouvement, Annals of Mathematics Studies, no. 17, Princeton University Press, 1947.

4. A. Wintner, Asymptotic distributions and infinite convolutions, Ann Arbor, Edwards Brothers, 1938.

5. - The analytical foundations of celestial mechanics, Princeton University Press, 1941.

Purdue University 